\title{
BMJ Open Protocol for studying cough frequency in people with pulmonary tuberculosis
}

\author{
Alvaro Proaño, ${ }^{1}$ Marjory A Bravard, ${ }^{2,3,4}$ Brian H Tracey, ${ }^{5}$ José W López, ${ }^{6,7}$ \\ German Comina, ${ }^{8,9}$ Mirko Zimic,,${ }^{7,10}$ Jorge Coronel, ${ }^{10}$ Gwenyth O'Neill Lee, ${ }^{8}$ \\ Luz Caviedes, ${ }^{10}$ Jose Luis Cabrera, ${ }^{11}$ Antonio Salas, ${ }^{12}$ Eduardo Ticona, ${ }^{13,14}$ \\ Daniela E Kirwan, ${ }^{15}$ Jon S Friedland, ${ }^{15,16}$ Carlton A Evans, ${ }^{4,15,16}$ \\ David A Moore, ${ }^{2,10,17}$ Robert H Gilman, ${ }^{2,10,18}$ Tuberculosis Working Group in Peru
}

To cite: Proaño A, Bravard MA, Tracey BH, et al. Protocol for studying cough frequency in people with pulmonary tuberculosis. BMJ Open 2016;6:e010365. doi:10.1136/bmjopen-2015010365

- Prepublication history and additional material is available. To view please visit the journal (http://dx.doi.org/ 10.1136/bmjopen-2015010365).

In memoriam LC who passed away in November 2012.

Received 26 October 2015 Revised 9 February 2016 Accepted 2 March 2016

\section{CrossMark}

For numbered affiliations see end of article.

Correspondence to Dr Robert H Gilman; rgilman@jhsph.edu / gilmanbob@gmail.com

\section{ABSTRACT}

Introduction: Cough is a key symptom of tuberculosis (TB) as well as the main cause of transmission. However, a recent literature review found that cough frequency (number of coughs per hour) in patients with TB has only been studied once, in 1969. The main aim of this study is to describe cough frequency patterns before and after the start of TB treatment and to determine baseline factors that affect cough frequency in these patients. Secondarily, we will evaluate the correlation between cough frequency and TB microbiological resolution.

Methods: This study will select participants with culture confirmed TB from 2 tertiary hospitals in Lima, Peru. We estimated that a sample size of 107 patients was sufficient to detect clinically significant changes in cough frequency. Participants will initially be evaluated through questionnaires, radiology, microscopic observation drug susceptibility broth TB-culture, auramine smear microscopy and cough recordings. This cohort will be followed for the initial 60 days of anti-TB treatment, and throughout the study several microbiological samples as well as $24 \mathrm{~h}$ recordings will be collected. We will describe the variability of cough episodes and determine its association with baseline laboratory parameters of pulmonary TB. In addition, we will analyse the reduction of cough frequency in predicting TB cure, adjusted for potential confounders.

Ethics and dissemination: Ethical approval has been obtained from the ethics committees at each participating hospital in Lima, Peru, Asociación Benéfica PRISMA in Lima, Peru, the Universidad Peruana Cayetano Heredia in Lima, Peru and Johns Hopkins University in Baltimore, USA. We aim to publish and disseminate our findings in peer-reviewed journals. We also expect to create and maintain an online repository for TB cough sounds as well as the statistical analysis employed.

\section{INTRODUCTION}

Tuberculosis (TB) is an infectious disease, and was responsible for 9.6 million new cases and 1.5 million deaths in $2014 .^{1}$ TB is transmitted in the air ${ }^{3}$ and cough is the most

\section{Strengths and limitations of this study}

- The algorithm employed in this project has been validated specifically for patients with pulmonary tuberculosis (TB), which enables us to use this algorithm in our patients.

- A strength of this project is that its results will reflect actual cough frequency episodes in pulmonary TB by utilising $24 \mathrm{~h}$ recordings in the patients' normal-day settings (traffic, dogs barking, etc). We expect that this will generate a novel method of evaluating cough in TB that can be used in real-world scenarios.

- Our study has the limitation that recordings have been processed through a semiautomated algorithm. To decrease time constraints, our longtime goal is to create a fully automated processing system. We anticipate that experience gained with semiautomated analysis will aid us in developing future algorithms.

important cause of transmission. ${ }^{4}$ Cough in people with pulmonary TB disease arises as a result of the inflammatory response to mycobacterial pulmonary infection. A reduction in cough is assumed to represent adequate response to treatment, and to result in decreased risk of spread of infection. Despite its crucial role in TB transmission, a recent literature review ${ }^{5}$ reported that cough frequency during TB therapy has not been studied since the work carried out by Loudon in the 1960s. ${ }^{6}{ }^{7}$ Thus, longitudinal cough frequency studies in TB are needed.

Loudon described cough frequency in $8 \mathrm{~h}$ overnight periods for 9 weeks. All sounds with amplitude and frequency consistent with possible cough events were recorded and then manually reviewed. ${ }^{8}$ His findings show a twofold reduction in the first 2 weeks of treatment, from a mean of 13.6 to 4.75 coughs $/ \mathrm{h}^{7}$ Mycobacterium tuberculosis colony forming units (CFU) also reduced significantly from $10^{6}$ at baseline to $10^{3} 2$ weeks 
later. ${ }^{9}{ }^{10}$ This evidence led to the idea that drugsusceptible patients with TB become sufficiently noninfective by the second week of treatment that they no longer pose a risk to others. This and other evidence led to the often-used policy that 2 weeks was the necessary duration of respiratory isolation for newly diagnosed patients started on appropriate treatment. Current evidence $^{11}$ and guidelines affirm this position; ${ }^{12-14}$ however, this 2-week policy has been criticised. ${ }^{15}{ }^{16}$ Our group has shown that drug-susceptible patients with TB remain sputum culture positive for longer. ${ }^{17}{ }^{18}$ Most importantly, the assumption that patients with TB are no longer coughing at 2 weeks has never been corroborated.

The 2015 CHEST guidelines recommend acoustic parameters to evaluate the frequency of cough. ${ }^{19}$ In order to ensure accurate measurement, it is important to use a standardised method such as automated cough counting with a validated algorithm. Despite the recently growing literature on this topic, these methods are principally being used in the field of non-infectious chronic disease. ${ }^{20-25}$ While algorithms for cough counting have been validated, ${ }^{26-30}$ our research protocol appears to be the first to do so specifically in patients with pulmonary TB. ${ }^{31} 32$

To address this knowledge gap, we have developed the Cayetano Cough Monitor (CayeCoM) and here describe a protocol for it to be used to study cough frequency in patients with pulmonary TB.

\section{METHODS}

\section{Study objectives}

The primary objective of this study is to describe cough frequency patterns in adults with pulmonary $\mathrm{TB}$ before and after treatment initiation.

The second objective of this study is to determine baseline characteristics that correlate with cough frequency, such as patient demographics, radiological findings, presence of multidrug-resistant TB (MDR-TB) and HIV status.

The third objective of this study is to test for an association between changes in cough frequency and microbiological resolution of TB disease during therapy.

\section{Study design}

This prospective cohort study will follow adult patients with pulmonary TB throughout their treatment period in Lima, Peru.

Participants with a confirmed or suspected diagnosis of active pulmonary TB will be referred to our study team. After obtaining written informed consent, we will record coughs prior to initiation of $\mathrm{TB}$ treatment. Participants will provide us with early morning sputum samples that will be tested for active pulmonary TB disease by testing at least one sputum sample using the microscopic observation drug susceptibility (MODS) broth culture assay ${ }^{33-35}$ and auramine smear microscopy to assess the bacillary load.
Patients in whom the pulmonary TB diagnosis is confirmed by MODS will receive treatment delivered by the National TB Programme as per standard practice. ${ }^{36}$ Figure 1 summarises the data to be collected at baseline and during the 60 days of follow-up.

\section{Study sites}

Peru has one of the highest TB incidence rates in the Americas. ${ }^{37}$ More than one-third of the incident TB cases in the Andean region are from Peru. With respect to rates of MDR-TB and extensively drug-resistant (XDR) TB, Peru ranks first in all of the Americas. However, under-reporting in the region may contribute to Peru's over-representation, as shown in the latest Pan American Health Organisation (PAHO) report. ${ }^{37}$

Within Peru, Lima and its metropolitan area account for most cases of MDR-TB and XDR-TB. ${ }^{38}$ Thus, we will recruit patients from two hospitals: Hospital Nacional Dos de Mayo (HNDM), located in the historic centre of Lima; and Hospital Nacional Daniel Alcides Carrión, located in Callao and which belongs to Lima's metropolitan area.

Our main site, HNDM, is a 650-bed teaching and public national tertiary referral hospital run by the Peruvian Ministry of Health (MINSA). It provides services to the poor population from the surrounding inner city area. HNDM is the only hospital in Peru with a negative pressure ward available for patients with TB. Our secondary site is another tertiary referral hospital run by MINSA, Hospital Nacional Daniel Alcides Carrión. This 462-bed teaching health facility lies in the Callao region.

\section{Study population}

The infectious disease and pulmonary physicians will refer participants to the research team. Criteria for referral are suspicion of active pulmonary TB or a confirmed case of active pulmonary TB which has not yet started treatment. Active pulmonary TB is defined by a positive MODS culture result. Participants will be excluded if they were $<18$ years of age, pregnant, have started a new treatment regimen for TB within the past 7 days, or are unable or unwilling to provide informed consent. If a patient changes the treatment regimen, for example, due to treatment failure or to an adverse drug reaction, this would also be considered as a new regimen. Pregnancy is defined by a positive result on the serum or urine $\beta$-human chorionic gonadotropin ( $\beta$-hCG) assay.

\section{Outcomes and case definitions}

The primary outcomes for this study are cough frequency and microbiological data from serial sputum samples. Cough frequency is defined as the number of cough episodes, or cough epochs, within a time period. Cough epochs are defined as cough events that are within a 2 s period frame. ${ }^{32}$

Regarding microbiological data, participants will be entered into the study if they have a positive culture 


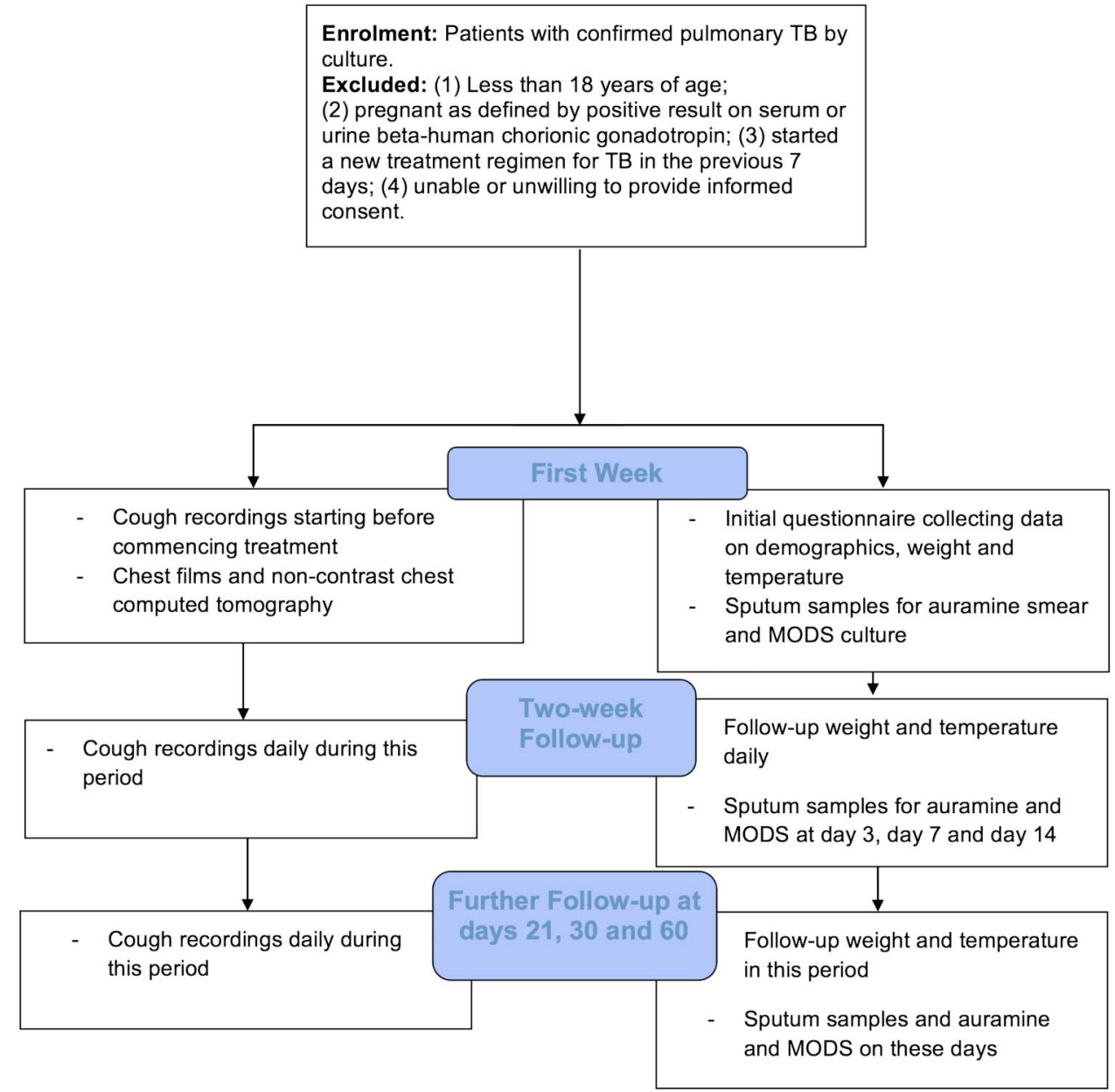

Figure 1 Flow diagram for CayeCoM study. CayeCoM, Cayetano Cough Monitor; MODS, microscopic observation drug susceptibility; TB, tuberculosis.

result. Treatment regimens will be adjusted as needed by the treating team based on the results of the MODS drug-susceptibility testing from their sputum. Our study team will not be involved in the treatment regimen selection.

Sputum smear conversion is defined as three consecutive smear-negative results, collected at least $8 \mathrm{~h}$ apart after initial smear positivity at diagnosis. ${ }^{12}$ Culture conversion is defined as two consecutive negative culture results, taken at least 30 days apart. This last definition is the one used in the MINSA ${ }^{36}$ and is recommended by the WHO. ${ }^{39}$ The date of conversion will be considered as the date of the first negative sputum smear or culture contributing to conversion.

Secondary outcomes include weight, temperature and radiological characteristics. When possible, radiological interpretation data from chest films and thoracic CT scans will be obtained. Chest X-ray films (CXR) provide a high negative predictive value for the presence of active $\mathrm{TB},{ }^{40}$ but $\mathrm{CXR}$ might be normal when in fact there is parenchymal disease. ${ }^{41}$ More specifically, CT scans correctly determine pulmonary $\mathrm{TB}$ cases in $91 \%$ of cases and CXR in only $49 \%$ of cases. ${ }^{41-44}$ In addition, CT scans provide higher sensitivity for the detection of lymphadenopathy, early bronchogenic spread and to evaluate cavitation and disease activity. ${ }^{44}$

\section{Sample size}

In a pilot study, we estimated that the frequency of cough in patients with TB before receiving treatment is approximately 327 coughs during a $24 \mathrm{~h}$ period with an SD of approximately 50. A sample size of 97 patients would enable us to detect a conservative decrease in the mean number of coughs in the $24 \mathrm{~h}$ period of at least 45 coughs after 2 weeks of treatment, with a $5 \%$ type I error probability and $80 \%$ power.

Under the hypothesis that patients with $\mathrm{TB}$ before treatment experience a high cough frequency, we hypothesise that after 2 weeks of anti-TB treatment, there will be a clinical response accompanied by a significant reduction in cough frequency. Response is defined as at least a twofold reduction in cough frequency, which was previously shown to occur within the first 2 weeks of treatment. ${ }^{7}$ For power calculations, it is assumed that all participants will eventually respond to treatment, according to our definition of response, and that once cough frequency has reduced in an individual it will not rise again. We assume that after the 2 weeks of 
treatment approximately $10 \%$ of patients would maintain a high frequency of cough. Thus, a sample size of 97 patients will allow us to detect an OR of at least 3.2 for the risk of patients not responding to TB treatment in 2 weeks of therapy, under a $95 \%$ significance and $80 \%$ power. An additional $10 \%$ of patients will be recruited to correct for patients who do not complete all of the study procedures. Thus, we will aim to recruit a total of 107 patients.

\section{Study organisation}

The Asociación Benéfica (A.B.) PRISMA and Universidad Peruana Cayetano Heredia in Lima, Peru will provide local administrative oversight. Overseas, oversight will be conducted by Johns Hopkins University in Baltimore, Maryland, USA.

In Lima, the Pampas office of A.B. PRISMA will provide operations and logistic support for fieldwork. An additional collaborating signal processing team will be based locally in the Universidad Nacional de Ingeniería, Lima, Peru, as well as at Tufts University, Massachusetts, USA.

Our collaborating biostatisticians are based at Tufts University, Tulane University and Universidad Peruana Cayetano Heredia, Lima, Peru. All investigators are involved in protocol design and technical support and will remain involved in the ongoing analyses.

\section{Personnel, training and logistics}

Nurses have been trained by study staff to obtain sputum samples in a best-practice fashion based on previous work, ${ }^{45}$ and to operate and troubleshoot all recorder devices, memory cards and battery packs. We will adhere to recommended infection prevention and control practices for TB to reduce biorisk in healthcare professionals and patients. ${ }^{47}$ Written informed consent is required prior to research participation. At the time of enrolment, participants will follow the procedures outlined in figure 1.

Participants with active pulmonary TB will be followed throughout their TB treatment. After the identification of active pulmonary TB and on the basis of convenience, participants who consent will undergo CXR and a noncontrast thoracic CT scan.

The first day of a new $\mathrm{TB}$ treatment regimen is defined as 'day 0'. An initial questionnaire will be completed on that day (see online supplementary file 1). It should be mentioned that we used a 5-level ordinal scale instead of 10 to make it simpler for our interviewees. We have found it easier in this setting for research participants to interpret 5-levels each with defining words (never, little, much, almost always and always) rather than 10. This questionnaire is similar to the one that was employed in a previous study. ${ }^{48}$ Baseline cough frequency will be obtained by performing an audio recording of the patients before they obtain their microbiological results, which is usually a few days prior to treatment initiation. Hence, participants will be recorded from at least one day prior to treatment and throughout their first two weeks of treatment. They will subsequently be recorded for $24 \mathrm{~h}$ on or around days 21 , 30 and 60 of treatment, although up to two days' date deviation for Sundays and public holidays will be allowed.

Recordings will start at 09:00 and will be as continuous as possible. Occasionally, incomplete recordings could be obtained due to malfunction of equipment or patient non-compliance. On the recording days, clinical data will be gathered, including: weight, temperature and sputum samples for smear and MODS results. The number of days to culture positivity in the MODS liquid culture assay will be recorded in order to assess the microbiological burden in the patients' samples, based on prior work done with a similar technique. ${ }^{49}$

\section{Audio recording}

Design of the audio recording equipment, the CayeCoM device, builds on previous chronic cough ambulatory audio recordings. ${ }^{27} 5051$ The CayeCoM device is a Marantz PMD 620 professional handheld recorder, using an Audio-Technica AT899 sub-mini microphone with an AT8537 microphone power module. The microphone will be attached at the patient's lapel as shown in figure 2. The recorder is adapted to work with an external lithium battery supply (Enix Energies 800040) to enable continuous $24 \mathrm{~h}$ recordings. The audio is recorded onto a SanDisk SDHC 8 GB card, at a sample rate of $48 \mathrm{kHz}$, encoding $64 \mathrm{kbps}$ in mono in MP3 format. The audio equipment is kept inside a basic pack connected to a lapel microphone. Batteries and SD cards will be exchanged daily by the study nurses. In pilot research, participants tolerated the audio equipment well, wearing them $24 \mathrm{~h}$ a day and taking them off only to bathe.

\section{Processing of audio recordings}

The recorded signals will be analysed after all patient recordings are completed. For cough analysis, software developed by our group and previously described in detail will be used. ${ }^{31} 32$ Thus, we provide only a brief review here and refer interested readers to our previous publications.

Briefly, cough recordings will be analysed using a two-step algorithm: first, event detection, followed by event classification into cough versus non-cough. Detection of acoustic events will be based on the signal energy proportional to the square of the voltage of the signal. An acoustic event will detect if the signal energy exhibited a rapid increase above a time-varying baseline estimate of ambient noise. The next stage of processing seeks to classify detected events. Here, the spectral features of each time frame in the acoustic event are characterised using Mel-frequency cepstral coefficients and their derivatives. As described in detail elsewhere, ${ }^{32}$ a training data set will be used to develop a classifier based on the sequential minimal optimisation (SMO) 


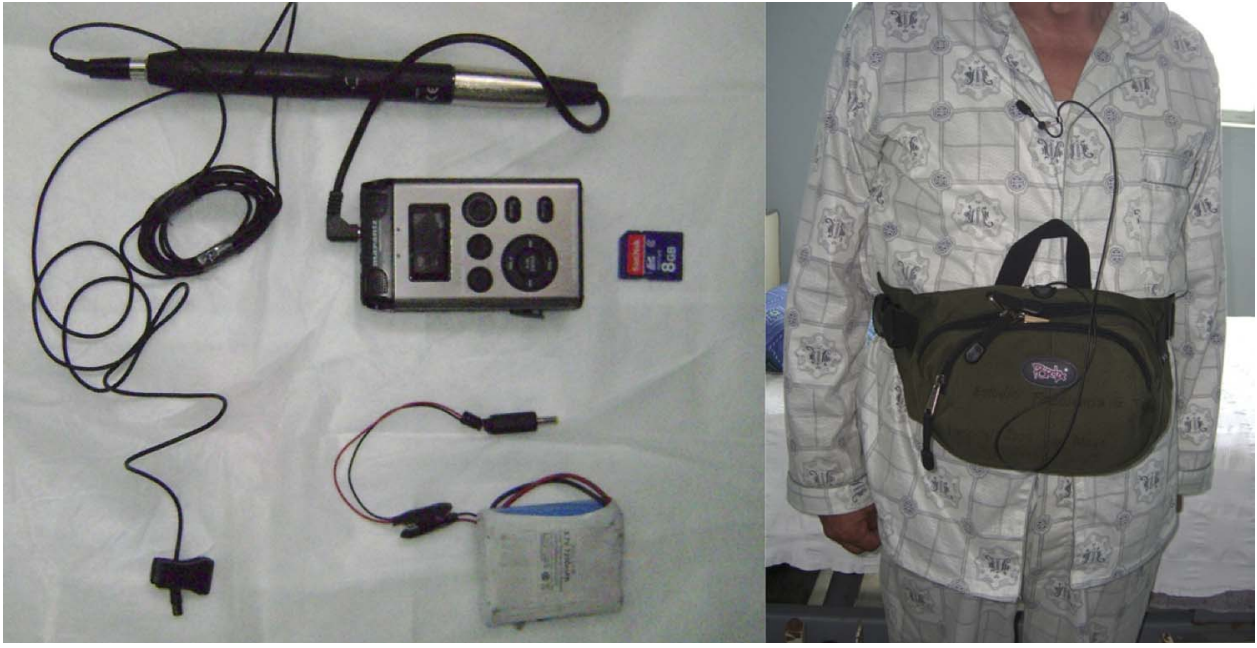

Figure 2 Picture of the Cayetano Cough Monitor (CayeCoM).

algorithm. On the basis of classifier outputs, each acoustic event will be marked as 'cough' or 'not-cough'.

Isolated cough events will be automatically combined into cough epochs, or bursts of closely spaced individual coughs, following previous research. ${ }^{52}$ We will employ a definition of cough epochs, as defined in the 'Outcomes and case definitions' section above. Note that within the cough literature, a variety of metrics are available for describing a cough, and there is no clear evidence as to which of them are most clinically meaningful. We have previously published a review and discussion of these various metrics (number of individual coughs, number of cough bouts or epochs, number of $1 \mathrm{~s}$ periods containing cough, etc).$^{32}$

We will employ a semiautomated approach in which cough epochs that are automatically detected will then be manually reviewed to eliminate false positives. This is necessary as our recordings will be made in very noisy environments (outside clinical settings) and false detection rates for a fully automated system remain high. For this study, a simple graphical user interface will be constructed to allow nurses to review automatically detected epochs, enabling them to listen to each as often as needed, and then to either accept or reject the detected cough. Thus, the review of automatically detected coughs acts to eliminate algorithmic falsepositive coughs.

Validation: The approach described in the above paragraphs was previously validated using as the gold standard a fully manual review of 60 files (15 participants, 4 randomly selected time periods per participant) in which two nurses listened to all files in their entirety. ${ }^{32}$ Since nurses only manually marked the start of each cough, validation was compared on the basis of the epoch definition described above. The semiautomated approach described above gave $75.5 \%$ sensitivity in detecting coughs (a true-positive rate of $6.8 / \mathrm{h}$ ) with an average false-positive rate of $0.5 / \mathrm{h} .{ }^{32}$ While the semiautomated approach does require time for human review, the initial automated step will remove the large majority of possible events. Thus, on average, review time is reduced by nearly two orders of magnitude compared with a fully manual review in which the entire recording is reviewed. We will also maintain the privacy of participants, as non-cough events, such as conversation, will never be reviewed by the human ear.

\section{Microbiology}

The microbiological tests will be carried out in a Biosafety Containment Level 3 research laboratory situated within Universidad Peruana Cayetano Heredia in Lima, Peru. The sputum samples will be digested and decontaminated by the standard $\mathrm{NaOH}-\mathrm{n}$-acetyl cysteine method. ${ }^{53}$ For smear microscopy, an aliquot of $100 \mu \mathrm{L}$ is stained with Auramine $\mathrm{O}$ and examined with $\times 400 \mathrm{mag}-$ nification. Results are determined as negative, paucibacillary (1-19 acid-fast bacilli (AFB) visualised in 40 fields), 1+ (20-199 AFB visualised in 40 fields), 2+ (5-50 AFB per field) and $3+(>50$ AFB per field). Culture and MODS susceptibility testing will be performed with the remaining samples, according to standard protocols. ${ }^{33-35}$

\section{Radiology}

Radiological information will be gathered when possible on a convenience basis. Priority will be given to CT scans, since they have been shown to be more sensitive in general. ${ }^{44}$ A previous study determined that the sensitivity for the prediction of active TB through CT scans was $96 \%$, whereas for CXR it was merely $48 \% .{ }^{54}$ Films will be read by a local radiologist and a US boardcertified radiologist blinded to the patient's demographics and outcomes. They will provide an interpretation that is standardised as per our study protocol to describe radiological findings including cavitation, consolidation, lymphadenopathy and effusions (see online supplementary file 2). We will explore whether these radiological findings are predictive of microbiological burden and cough frequency. 
Cavitations will be further described by the size, location, presence or absence of an air-fluid level, and cavity wall thickness based on prior work that shows the relevance of these findings to pulmonary $\mathrm{TB}$ and, most importantly, to infectivity. ${ }^{75-58}$ It is therefore important to determine cavitations, and as Im et a $\tilde{l}^{\tilde{5}}$ have shown, CT correctly identifies cavitations in $58 \%$ of cases, whereas CXR only identifies $22 \%$.

\section{Statistical methodology and analysis}

All questionnaire data will be double digitised from paper forms using Visual FoxPro 9 Service Pack 2 (Microsoft Corp. Redmond, Washington, USA) and microbiological data will be double entered using Microsoft Access 2010 (Microsoft Corp. Redmond, Washington, USA). These two data sets will be crosscompared for validity and errors. From these data, descriptive statistics will be tabulated and graphed.

Cough analysis processing results will be stored as Matlab (Mathworks, Inc, Natick, Massachusetts, USA) files containing information regarding each event and its timestamp. Algorithmically detected coughs will be annotated in the files. After manual review, isolated cough events will be grouped into cough epochs, or bursts of closely spaced individual coughs within $2 \mathrm{~s}$, following published work on cough evaluation. ${ }^{52}$

For the first study objective of describing cough frequency, cough epochs will be plotted throughout the day, and cough frequency will be summarised as the frequency of cough epochs per hour. Positively skewed cough data may be log-transformed to facilitate data visualisation and analysis. To address the second study objective, correlation of characteristics with cough frequency, we will use generalised estimating equations (GEE) based Poisson or negative binomial regression with baseline microbiological status, and trigonometric (sine/cosine) terms to model circadian periodicity, as the independent variables. In addition, a multiple logistic regression in a longitudinal generalised linear model (GLM) framework analysis will evaluate a function of sputum bacillary load and with cough frequency that we propose as a potential predictor of TB transmissibility. In all cases, we will correct for outliers, and nested models will be compared using the likelihood ratio test. We will also consider variables such as gender, HIV status, drug resistance and history of TB in our analysis, either by stratifying or by adjusting for these variables in our models.

To test the association between cough frequency and microbiological resolution of TB disease associated with the third aim of this study, time-to-event survival analyses where the outcomes of interest are sputum smear conversion, and culture conversion, as defined above, and the primary predictors of interest are cough frequency at baseline, during treatment, and time to twofold reduction in cough frequency. In addition, secondary analyses of weight, temperature and radiological characteristics will be conducted using GLMs and GEE logistic regression as appropriate.

\section{Dissemination}

Written informed consent will be obtained from all participants. Test results will be delivered by telephone or at subsequent visits at which time a team physician or nurse will be able to explain the results to the study participants. TB treatment remains the responsibility of the medical staff in charge and the National TB Programme. We aim to publish and disseminate our results once the project is complete. We also expect to create and maintain an online repository for TB cough sounds as well as the statistical analysis employed.

\section{DISCUSSION}

We will determine cough frequency before and during anti-TB treatment using the CayeCoM device. We will identify baseline predictors of cough frequency during TB treatment and evaluate the correlation between change in cough frequency and microbiological resolution.

The medical literature currently lacks information about cough frequency in TB. As recently noted by Turner and Bothamley, ${ }^{5}$ cough frequency in patients undergoing TB treatment has only been studied once, almost half a century ago. ${ }^{6}$ This previous study has the limitation of only being conducted within an $8 \mathrm{~h}$ period, overnight, and thus there is no information on daytime coughing or the effect of the diurnal rhythm on cough. A similar study ${ }^{58}$ demonstrated that the severity of cough and pathological chest X-ray findings were associated with higher levels of TB transmission. However, their study did not measure cough frequency but instead focused on a participantive characteristic: cough severity. It should be noted that to assess cough frequency, one must utilise objective acoustic parameters, since selfreported cough is unreliable. ${ }^{19}$ As reported in abstract form, the objective acoustic Leicester Cough Monitor (LCM) has been used to evaluate $24 \mathrm{~h}$ cough recordings in patients with pulmonary TB before starting treatment, showing that cough frequency is reduced at night. ${ }^{59}$ This further justifies re-evaluation of Loudon's overnight study.

Our project has several strengths and limitations. An important strength is the generation of $24 \mathrm{~h}$ cough recordings, which will provide lengthy recordings, will enable evaluation of cough patterns at different times of day, and also have the benefit of being recorded during a normal day in real-world settings where we expect our device to be used in the future. Normal day recordings are confounded by background noise, which is a challenge for analysis of cough recordings, considering that traffic and environmental noise (such as dogs barking, music and television) may generate noises similar to cough. To diminish this effect, we have incorporated a time-varying estimate of the noise background as well as a data quality control. Having a semiautomated algorithm is a limitation, since it requires time and human input, as well as a strength since the human ear is the 
gold standard for determining the characteristic sound of cough. Similar to Loudon and Romans' ${ }^{8}$ proposal, our algorithm will help to screen and reduce the length of the recordings to $\sim 5 \%$ of their original length, without affecting sensitivity and improving specificity. ${ }^{32}$ In the long term, we aim to improve our algorithm (ie, fully automated processing), and we anticipate that experience gained with semiautomated analysis will aid us in this process. In addition, we are now developing second-generation devices where the validity is improved by employing accelerometers. This study is limited by restriction to only non-pregnant adults because this is the population for which the algorithm has been validated. However, future research is planned to include these important vulnerable populations.

CayeCoM has been validated for $24 \mathrm{~h}$ recordings, ${ }^{32}$ whereas PulmoTrack (PulmoTrack-CC, KarmelSonix, Haifa, Israel) was validated for $25 \mathrm{~min}^{29}$ and the Hull Automatic Cough Counter for $1 \mathrm{~h}$ recordings. ${ }^{27}$ Other systems have also validated their algorithms for $24 \mathrm{~h}$ recordings, such as the LCM, ${ }^{28}{ }^{60}$ VitaloJAK $^{30}$ and the LifeShirt System. ${ }^{26}$ However, in contrast to our study, none of these algorithms have been validated either for pulmonary TB or within real-life settings (eg, traffic). We expect that this project will generate a novel method to evaluate treatment response. In future studies, we intend to better assess infectiousness by additionally quantifying TB in cough-generated aerosols.

Cough frequency should provide additional information regarding the evolution of the patients' medical condition. If a correlation with bacteriological treatment response is demonstrated, then this would have the potential to contribute to patient management without relying on a laboratory in adult patients with pulmonary TB. However, we should be careful when monitoring patients with TB since some may worsen after an initial positive response to therapy. It could assist with decisions regarding the need for the ongoing respiratory isolation of patients, treatment duration and identification of patients with treatment failure who may need modification of their treatment regimens. The device also has the potential to be used remotely, as in telemedicine. This is potentially important in a country such as Peru, where the majority of doctors live in the capital, leaving most of the country without a physician in their region. Cough monitoring devices seem challenging; however, we believe that this is the first step towards telemedicine in cough-TB. In Peru, many rural areas do not have facilities for laboratory diagnosis, but have at least one physician or healthcare professional. They may be trained in placing these devices. We are also working on making devices smaller, cheaper and easier to use.

\footnotetext{
Author affiliations

${ }^{1}$ Facultad de Medicina 'Alberto Hurtado', Universidad Peruana Cayetano Heredia, Lima, Perú

${ }^{2}$ Asociación Benéfica PRISMA, Lima, Perú
}

${ }^{3}$ Department of General Internal Medicine, Massachusetts General Hospital, Boston, Massachusetts, USA

${ }^{4}$ Innovation For Health And Development (IFHAD), Laboratory of Research and Development, Universidad Peruana Cayetano Heredia, Lima, Peru

${ }^{5}$ Department of Electrical and Computer Engineering, Tufts University,

Medford, Massachusetts, USA

${ }^{6}$ Instituto Nacional de Salud del Niño San Borja, Lima, Perú

${ }^{7}$ Laboratorio de Bioinformática y Biología Molecular, Facultad de Ciencias y

Filosofía, Universidad Peruana Cayetano Heredia, Lima, Perú

${ }^{8}$ Department of Global Community Health and Behavioral Sciences, Tulane University, New Orleans, Louisiana, USA

${ }^{9}$ Escuela Profesional de Ingeniería Física, Facultad de Ciencias, Universidad Nacional de Ingeniería, Lima, Perú

${ }^{10}$ Laboratorio de Investigación en Enfermedades Infecciosas, Laboratorio de Investigación y Desarrollo, Facultad de Ciencias y Filosofía, Universidad Peruana Cayetano Heredia, Lima, Perú

${ }^{11}$ Servicio de Neumología, Hospital Nacional Alcides Carrión, Lima, Perú

${ }^{12}$ Servicio de Neumología, Hospital Nacional Dos de Mayo, Lima, Perú

${ }^{13}$ Facultad de Medicina, Universidad Nacional Mayor de San Marcos, Lima, Perú

${ }^{14}$ Servicio de Enfermedades Infecciosas y Tropicales, Hospital Nacional Dos de Mayo, Lima, Perú

${ }^{15}$ Infectious Diseases \& Immunity, Imperial College London, London, UK

${ }^{16}$ Wellcome Trust Imperial College Centre for Global Health Research,

London, UK

${ }^{17}$ TB Centre, London School of Hygiene and Tropical Medicine, London, UK

${ }^{18}$ Program in Global Disease Epidemiology and Control, Department of International Health, Bloomberg School of Public Health, Johns Hopkins University, Baltimore, Maryland, USA

\section{Twitter Follow Alvaro Proaño at @proanoA and José López at @jolopezr}

Collaborators Tuberculosis Working Group in Peru. Other members of the Tuberculosis Working Group in Peru include Patricia Fuentes and Patricia Sheen (Universidad Peruano Cayetano Heredia, Lima, Peru); Aldo Vivar (Hospital Nacional Arzobispo Loayza, Lima, Peru); Eduardo Sanchez (Hospital Nacional Hipólito Unanue, Lima, Peru); Richard Rodríguez and María Prado (Hospital María Auxiliadora, Lima, Peru); Jesus Chacaltana (Hospital Nacional Daniel Alcides Carrion, Lima, Peru); Felix Llanos and Marco Ñavincopa (Hospital Nacional Dos De Mayo, Lima, Peru); Lilia Cabrera and Marco Varela (Asociación Benéfica PRISMA, Lima, Peru); Jorge Gustavo Hernández and Richard Oberhelman (Tulane University, New Orleans, USA); Roderick Escombe and Louis Grandjean (Imperial College London, London, UK); Jose Gomez-Marquez (Massachusetts Institute of Technology, Massachusetts, USA); Sumona Datta (IFHAD: Innovation For Health And Development at the Universidad Peruana Cayetano Heredia and at Imperial College London); David Bui (University of Arizona, Tucson, USA) and nurses from the Peruvian National TB Programme.

Contributors All authors were involved in the study design and writing of the manuscript, and all reviewed the final manuscript before submission. $M A B$ and JWL directly contributed to the study design and were responsible for supervision of data gathering. AP, BHT, JWL, MZ and GOL were responsible for data management and statistical analysis for this project.

Funding This work was funded in part by the National Institutes of Health award 5D43TW006581 'Infectious Diseases Training Program in Peru', award 5D43TW009349-03 'Inter-American Training for Innovations in Emerging Infectious Diseases', Grand Challenges Canada Contract No. 0539-01-10 'Smartphone app for cough monitoring of tuberculosis patients', and award 5R21Al094143-02 'Cough—a rapid indicator of response to therapy in pulmonary TB'. CAE and JSF thank the Imperial College Biomedical Research Centre for financial support. The contributions of CAE to this research were funded by: the Joint Global Health Trials consortium of the Wellcome Trust, UK-MRC and DFID (award MR/K007467/1); The Wellcome Trust (awards 078340/Z/05/Z, 105788/Z/14/Z and 201251/Z/16/Z); The Bill and Melinda Gates Foundation award OPP1118545; and IFHAD: Innovation For Health And Development.

Competing interests None declared. 
Ethics approval Each participating hospital in Lima, Peru, Asociación Benéfica PRISMA in Lima, Peru, the Universidad Peruana Cayetano Heredia in Lima, Peru and Johns Hopkins University in Baltimore, USA.

Provenance and peer review Not commissioned; externally peer reviewed.

Data sharing statement No unpublished data are available. We aim to publish and disseminate our results once the project is complete. We also expect to create and maintain an online repository for TB cough sounds as well as the statistical analysis employed.

Open Access This is an Open Access article distributed in accordance with the terms of the Creative Commons Attribution (CC BY 4.0) license, which permits others to distribute, remix, adapt and build upon this work, for commercial use, provided the original work is properly cited. See: http:// creativecommons.org/licenses/by/4.0/

\section{REFERENCES}

1. World Health Organization. Global tuberculosis report 2015. Geneva, Switzerland: World Health Organization, 2015.

2. Riley RL, Mills CC, Nyka W, et al. Aerial dissemination of pulmonary tuberculosis: a two-year study of contagion in a tuberculosis ward. Am J Epidemiol 1959;70:185-96.

3. Riley RL, Mills CC, O'Grady F, et al. Infectiousness of air from a tuberculosis ward. Ultraviolet irradiation of infected air: comparative infectiousness of different patients. Am Rev Respir Dis 1962;85:511-25.

4. Loudon RG, Roberts RM. Singing and the dissemination of tuberculosis. Am Rev Respir Dis 1968:98:297-300.

5. Turner RD, Bothamley GH. Cough and the transmission of tuberculosis. J Infect Dis 2015;211:1367-72.

6. Loudon RG, Brown LC. Cough frequency in patients with respiratory disease. Am Rev Respir Dis 1967;96:1137-43.

7. Loudon RG, Spohn SK. Cough frequency and infectivity in patients with pulmonary tuberculosis. Am Rev Respir Dis 1969;99:109-11.

8. Loudon RG, Romans WE. Cough-monitoring equipment. Med Res Eng 1967;6:25-7.

9. Jindani A, Aber VR, Edwards EA, et al. The early bactericidal activity of drugs in patients with pulmonary tuberculosis. Am Rev Respir Dis 1980;121:939-49.

10. Mitchison DA. Infectivity of patients with pulmonary tuberculosis during chemotherapy. Eur Respir J 1990;3:385-6.

11. Datta S, Sherman JM, Bravard MA, et al. Clinical evaluation of tuberculosis viability microscopy for assessing treatment response. Clin Infect Dis 2015;60:1186-95.

12. American Thoracic Society, Centers for Disease Control and Prevention, Infectious Diseases Society of America. American Thoracic Society/Centers for Disease Control and Prevention/ Infectious Diseases Society of America: controlling tuberculosis in the United States. Am J Respir Crit Care Med 2005;172: 1169-227

13. Jensen PA, Lambert LA, lademarco MF, et al. CDC. Guidelines for preventing the transmission of Mycobacterium tuberculosis in health-care settings, 2005. MMWR Recomm Rep 2005;54:1-141.

14. Internal Clinical Guidelines Team (UK). Tuberculosis: Prevention, Diagnosis, Management and Service Organisation. London: Nationa Institute for Health and Care Excellence (UK), 2011 Jan. (NICE Guideline, No. 33.) Available from: http://www.ncbi.nlm.nih.gov/ books/NBK338750/

15. Noble RC. Infectiousness of pulmonary tuberculosis after starting chemotherapy. Am J Infect Control 1981;9:6-10.

16. Sepkowitz KA. How contagious is tuberculosis? Clin Infect Dis 1996;23:954-62.

17. Kawai V, Soto G, Gilman $\mathrm{RH}$, et al. Tuberculosis mortality, drug resistance, and infectiousness in patients with and without HIV infection in Peru. Am J Trop Med Hyg 2006;75:1027-33.

18. Fitzwater SP, Caviedes L, Gilman RH, et al. Prolonged infectiousness of tuberculosis patients in a directly observed therapy short-course program with standardized therapy. Clin Infect Dis 2010;51:371-8.

19. Boulet LP, Coeytaux RR, McCrory DC, et al. Tools for assessing outcomes in studies of chronic cough: CHEST guideline and expert panel report. Chest 2015;147:804-14.

20. Pavesi L, Subburaj S, Porter-Shaw K. Application and validation of a computerized cough acquisition system for objective monitoring of acute cough: a meta-analysis. Chest 2001;120:1121-8.

21. Smith J, Woodcock A. New developments in the objective assessment of cough. Lung 2008;186(Suppl 1):S48-54.
22. Yousaf N, Monteiro W, Parker D, et al. Long-term low-dose erythromycin in patients with unexplained chronic cough: a double-blind placebo controlled trial. Thorax 2010;65:1107-10.

23. Ryan NM, Birring SS, Gibson PG. Gabapentin for refractory chronic cough: a randomised, double-blind, placebo-controlled trial. Lancet 2012;380:1583-9.

24. Koehler U, Brandenburg U, Weissflog A, et al. [LEOSound, an innovative procedure for acoustic long-term monitoring of asthma symptoms (wheezing and coughing) in children and adults] Pneumologie 2014;68:277-81.

25. Sterling $\mathrm{M}$, Rhee $\mathrm{H}$, Bocko $\mathrm{M}$. Automated cough assessment on a mobile platform. J Med Eng 2014;2014:pii: 951621.

26. Coyle MA, Keenan DB, Henderson LS, et al. Evaluation of an ambulatory system for the quantification of cough frequency in patients with chronic obstructive pulmonary disease. Cough 2005;1:3.

27. Barry SJ, Dane AD, Morice $\mathrm{AH}$, et al. The automatic recognition and counting of cough. Cough 2006;2:8.

28. Birring SS, Fleming T, Matos S, et al. The Leicester Cough Monitor: preliminary validation of an automated cough detection system in chronic cough. Eur Respir J 2008;31:1013-18.

29. Vizel E, Yigla M, Goryachev $Y$, et al. Validation of an ambulatory cough detection and counting application using voluntary cough under different conditions. Cough 2010;6:3.

30. Barton A, Gaydecki P, Holt K, et al. Data reduction for cough studies using distribution of audio frequency content. Cough 2012;8:12.

31. Tracey $\mathrm{BH}$, Comina $\mathrm{G}$, Larson $\mathrm{S}$, et al. Cough detection algorithm for monitoring patient recovery from pulmonary tuberculosis. Conf Proc IEEE Eng Med Biol Soc 2011;2011:6017-20.

32. Larson S, Comina G, Gilman $\mathrm{RH}$, et al. Validation of an automated cough detection algorithm for tracking recovery of pulmonary tuberculosis patients. PLOS ONE 2012;7:e46229.

33. Caviedes L, Lee TS, Gilman RH, et al. Rapid, efficient detection and drug susceptibility testing of Mycobacterium tuberculosis in sputum by microscopic observation of broth cultures. J Clin Microbiol 2000;38:1203-8.

34. Moore DA, Mendoza D, Gilman RH, et al. Microscopic observation drug susceptibility assay, a rapid, reliable diagnostic test for multidrug-resistant tuberculosis suitable for use in resource-poor settings. J Clin Microbiol 2004;42:4432-7.

35. Moore DA, Evans CA, Gilman RH, et al. Microscopic-observation drug-susceptibility assay for the diagnosis of TB. N Engl J Med 2006;355:1539-50.

36. Ministerio de Salud del Perú. Norma Técnica de Salud Para la Atención Integral de las Personas Afectadas por Tuberculosis. Lima Perú, 2013.

37. Tuberculosis in the Americas: Regional Report 2012. Epidemiology, control and financing. Washington DC: Pan American Health Organization, 2013

38. Bonilla CA, Crossa A, Jave HO, et al. Management of extensively drug-resistant tuberculosis in Peru: cure is possible. PLOS ONE 2008;3:e2957.

39. World Health Organization. Definitions and reporting framework for tuberculosis-2013 revision. Geneva, Switzerland: World Health Organization, 2013.

40. Bhalla AS, Goyal A, Guleria R, et al. Chest tuberculosis: radiological review and imaging recommendations. Indian J Radiol Imaging 2015;25:213-25.

41. Jeong YJ, Lee KS. Pulmonary tuberculosis: up-to-date imaging and management. AJR Am J Roentgenol 2008;191:834-44.

42. Woodring $\mathrm{JH}$, Vandiviere $\mathrm{HM}$, Fried $\mathrm{AM}$, et al. Update: the radiographic features of pulmonary tuberculosis. AJR $A m \mathrm{~J}$ Roentgenol 1986;146:497-506.

43. Lee KS, Hwang JW, Chung MP, et al. Utility of CT in the evaluation of pulmonary tuberculosis in patients without AIDS. Chest 1996;110:977-84.

44. Skoura E, Zumla A, Bomanji J. Imaging in tuberculosis. Int J Infect Dis 2015;32:87-93.

45. Alisjahbana B, van Crevel R, Danusantoso $\mathrm{H}$, et al. Better patient instruction for sputum sampling can improve microscopic tuberculosis diagnosis. Int J Tuberc Lung Dis 2005;9:814-17.

46. Khan MS, Dar O, Sismanidis C, et al. Improvement of tuberculosis case detection and reduction of discrepancies between men and women by simple sputum-submission instructions: a pragmatic randomised controlled trial. Lancet 2007;369:1955-60.

47. WHO Policy on TB Infection Control in Health-Care Facilities, Congregate Settings and Households. Geneva: World Health Organization; 2009. Available from: http://www.ncbi.nlm.nih.gov/ books/NBK179249/ 
48. Rocha C, Montoya R, Zevallos K, et al. The Innovative Socioeconomic Interventions Against Tuberculosis (ISIAT) project: an operational assessment. Int J Tuberc Lung Dis 2011;15(Suppl 2): S50-7.

49. Carroll NM, Uys P, Hesseling A, et al. Prediction of delayed treatment response in pulmonary tuberculosis: use of time to positivity values of Bactec cultures. Tuberculosis (Edinb) 2008:88:624-30.

50. Paul IM, Wai K, Jewell SJ, et al. Evaluation of a new self-contained, ambulatory, objective cough monitor. Cough 2006;2:7.

51. Matos S, Birring SS, Pavord ID, et al. An automated system for 24-h monitoring of cough frequency: the Leicester Cough Monitor. IEEE Trans Biomed Eng 2007;54:1472-9.

52. Kelsall A, Decalmer S, Webster D, et al. How to quantify coughing correlations with quality of life in chronic cough. Eur Respir $J$ 2008;32:175-9.

53. Kent PT, Kubica GP, CDC. Public health mycobacteriology: a guide for the level III laboratory. U.S. Department of Health and Human Services, Public Health Service, Centers for Disease Control, 1988
54. Raniga S, Parikh N, Arora A, et al. Is HRCT reliable in determining disease activity in pulmonary tuberculosis? Indian J Radiol Imaging 2006;16:221-8.

55. Im JG, Itoh $\mathrm{H}$, Shim $\mathrm{YS}$, et al. Pulmonary tuberculosis: $\mathrm{CT}$ findings -early active disease and sequential change with antituberculous therapy. Radiology 1993;186:653-60.

56. Rodrigo T, Cayla JA, Garcia de Olalla P, et al. Characteristics of tuberculosis patients who generate secondary cases. Int J Tuberc Lung Dis 1997;1:352-7.

57. Van Dyck P, Vanhoenacker FM, Van den Brande P, et al. Imaging of pulmonary tuberculosis. Eur Radiol 2003;13:1771-85.

58. Jones-Lopez EC, Kim S, Fregona G, et al. Importance of cough and $M$. tuberculosis strain type as risks for increased transmission within households. PLOS ONE 2014;9:e100984.

59. Turner R, Repossi A, Matos S, et al. S79 cough prevalence and frequency in pulmonary tuberculosis. Thorax 2014;69(Suppl 2): A43-4.

60. Yousaf N, Monteiro W, Matos S, et al. Cough frequency in health and disease. Eur Respir J 2013;41:241-3. 


\section{Correction}

Proaño A, Bravard MA, Tracey BH, et al. Protocol for studying cough frequency in people with pulmonary tuberculosis. BMJ Open 2016;6:e010365. The segmentation of the eighth author's name is incorrect. This author's last name is Lee, and middle name is $\mathrm{O}^{\prime} \mathrm{Neill}$.

BMJ Open 2016;6:e010365corr1. doi:10.1136/bmjopen-2015-010365corr1

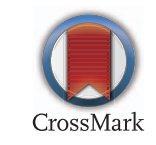




\section{Correction}

Proaño A, Bravard MA, Tracey BH, et al. Protocol for studying cough frequency in people with pulmonary tuberculosis. BMJ Open 2016;6:e010365. The segmentation of the tenth author's name is incorrect: his first name is Jose, middle name is Luis and last name is Cabrera, and he should be cited as Cabrera JL.

BMJ Open 2016;6:e010365corr2. doi:10.1136/bmjopen-2015-010365corr2 